\title{
SUSCEPTIBILITY TO ANTIBIOTICS IN URINARY TRACT INFECTIONS IN A SECONDARY CARE SETTING FROM 2005-2006 AND 2010-2011, IN SÃO PAULO, BRAZIL: DATA FROM 11,943 URINE CULTURES
}

Érique José PEIXOTO DE MIRANDA(1), Gerson Sobrinho SALVADOR DE OLIVEIRA(1), Felício Lopes ROQUE(2), Sílvia Regina DOS SANTOS(3), Rodrigo Diaz OLMOS(4) \& Paulo Andrade LOTUFO(5)

\begin{abstract}
SUMMARY
Introduction: Urinary tract infection (UTI) has a high incidence and recurrence, therefore, treatment is empirical in the majority of cases. Objectives: The aim of this study was to analyze the urine cultures performed at a secondary hospital, during two periods, 2005-2006 and 2010-2011, and to estimate the microbial resistance. Patients and methods: We analyzed 11,943 aerobic urine cultures according to basic demographic data and susceptibility to antibiotics in accordance with the Clinical and Laboratory Standards Institute (CLSI) for Vitek 1 and 2. Results: Most of our cohort consisted of young adult females that were seen at the Emergency Department. E. coli was the most frequent (70.2\%) among the 75 species isolated. Resistance of all isolates was $\geq 20 \%$ for trimethoprim/sulfamethoxazole (TMP/SMX), norfloxacin, nitrofurantoin, cefazolin and nalidixic acid. Although $E$. coli was more susceptible (resistance $\geq 20 \%$ for TMP/SMX and nalidixic acid) among all of the isolates, when classified by the number and percentage of antibiotic resistance. Global resistance to fluoroquinolones was approximately $12 \%$. Risk factors for $E$. coli were female gender and an age less than 65 years. Men and patients older than 65 years of age, presented more resistant isolates. Extended spectrum beta-lactamases (ESBL) were identified in 173 out of 5,722 Gram-negative isolates (3.0\%) between 2010 and 2011. Conclusion: $E$. coli was the most frequent microbe isolated in the urine cultures analyzed in this study. There was a significant evolution of bacterial resistance between the two periods studied. In particular, the rise of bacterial resistance to fluoroquinolones was concerning.
\end{abstract}

KEYWORDS: Urinary tract infection; Bacteria; Susceptibility test; Urine culture; Brazil.

\section{INTRODUCTION}

Urinary tract infection (UTI) is one of the most common infections diagnosed in patients, ranking first in the United States of America (USA). UTIs are characterized by high incidence, high recurrence and a mild severity of evolution in most cases. Because of the uncomfortable symptoms they cause, they often force physicians to introduce antibiotic treatment prior to obtaining the culture results ${ }^{14,15}$. The resolution of between $25 \%$ and $42 \%$ of cystitis UTIs in the placebo arms of randomized controlled trials, with little incidence of evolution to complicated infections and a proportion of one case of pyelonefritis per every 28 cases of cystitis, confirms the mild character of UTIs ${ }^{15}$. Current guidelines establish that lower UTIs in young women should be treated empirically with short-term antibiotics. Therefore, it is important that the antimicrobial susceptibility data of major uropathogens are known.
In particular, given that it is an infection that is most often treated empirically, data on major uropathogens should be systematically and regularly analyzed. However, in Brazil, there is a paucity of such data.

Despite the guidelines, requests for urinalysis and microscopic examination of urine (pyuria, leukocyte esterase, nitrite, and the presence of bacteria) as well as requests for urine culture for diagnosis of uncomplicated UTIs, is common among physicians in Brazil ${ }^{14,23}$. As a result, many cases that should be treated in a primary setting care are unnecessarily referred to the emergency department of secondary hospitals, resulting in increased health care costs. In clinical practice, physicians and their patients commonly insist that urine tests are necessary for the diagnosis of uncomplicated UTIs. The greatest proof of these assertions is evident from retrospective studies in several

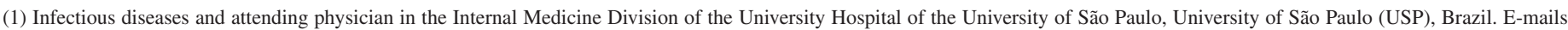
eriquemiranda@gmail.com; gersonsalvador@gmail.com.

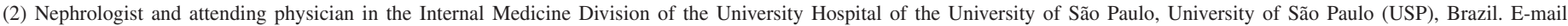
felicioroque@uol.com.br.

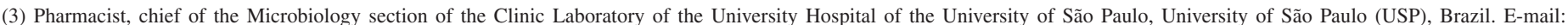
silviados@uol.com.br

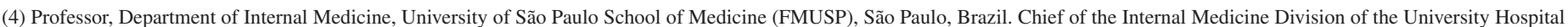
of the University of São Paulo, University of São Paulo (USP), Brazil. E-mail: olmos.rodrigo@gmail.com.

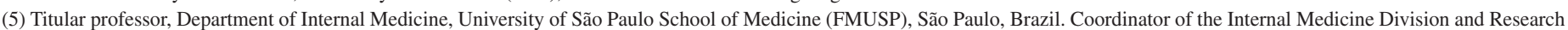
Mayor of the University Hospital of the University of São Paulo, University of São Paulo (USP), Brazil. E-mail: palotufo@ hu.usp.br.

Sponsorship: None.

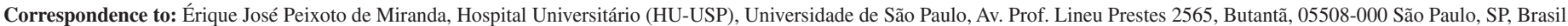
Tel.: +55 11 3091-9275. E-mail: eriquemiranda@gmail.com. 
PEIXOTO DE MIRANDA, E.J.; SALVADOR DE OLIVEIRA, G.S.; ROQUE, F.L.; DOS SANTOS, S.R.; OLMOS, R.D. \& LOTUFO, P.A. - Susceptibility to antibiotics in urinary tract infections in a secondary care setting from 2005-2006 and 2010-2011, in São Paulo, Brazil: data from 11,943 urine cultures. Rev. Inst. Med. Trop. Sao Paulo, 56(4): 313-24, 2014.

regions of Brazil, which were only possible because of this "breach of protocol" ${ }^{\prime 2,4,5,9,10,19,20,22,24,25,26}$.

We hypothesized that, in recent years in our environment, the microbiological profile of isolated uropathogens has become increasingly worse. Recent literature ${ }^{15}$ postulates a fluoroquinolone sparing protocol; however, if more than $20 \%$ of uropathogens are resistant to a drug, it cannot be used empirically. Therefore, the aim of this study is to retrospectively analyze the bacterial susceptibility data of uropathogens isolated from patients at a university community hospital in the last decade and to establish a new treatment protocol in this healthcare setting.

\section{MATERIALS AND METHODS}

The University Hospital of São Paulo is a 246-bed secondary care setting, one of the teaching hospitals of the University of São Paulo School of Medicine, and serves a population of approximately 500,000 inhabitants in the western area of the city of São Paulo. The hospital Emergency Department serves approximately 800 patients a day.

This is a retrospective study, which included positive urine cultures from patients who were collected during the periods from 2005 to 2006 and from 2010 and 2011, at the University Hospital in the Emergency Department, Outpatient Clinics, and from external patients. The aim of the study was to compare resistance to antimicrobial agents during two time periods, regardless of whether more than one urine culture per patient was collected during those time periods. The samples were processed and analyzed at the Section of Microbiology of the Clinical Laboratory of the University Hospital.

Only aerobic bacterial infections were analyzed in this study. Anaerobic bacterial infection, fungal infection and negative cultures were excluded from this study. Positive urine cultures were obtained by consulting Apolo software (Oracle Corporation, Redwood City, California, USA), without consulting the medical records of the patients, except for information about patients with urine culture with count $<$ 100,000 colony forming units (CFU) per milliliter of urine.

Urine collected by all methods during these time periods was included, although the standardized laboratory method is to collect the specimen urine midstream.

Urine cultures were taken quantitatively distributed on blood agar and MacConkey agar plates with a calibrated loop with a volume of $10 \mu \mathrm{L}$ (references 35095 and 35092, respectively, bioMérieux, Rio de Janeiro, Brazil). CPS ID2 chromogenic agar (bioMérieux, Rio de Janeiro, Brazil) has been used in the laboratory since 2012. Urine cultures with a count $\geq 100,000$ colony forming units (CFU) per milliliter of urine were considered positive after incubation in a bacteriologic incubator at 35 ${ }^{\circ} \mathrm{C}$ for at least 24 hours ${ }^{27}$. This study also included as positive counts $>$ $1,000 \mathrm{CFU}$ and $<100,000 \mathrm{CFU}$ of less than or equal to two infectious agents isolated (only pure or mixed culture) in the analysis because the patients presented symptoms of a UTI, which was documented in their chart $^{16}$. Only one the most significant germs isolated between two agents from each agar plate was considered in the analysis.

Identification of bacterial species and an antibiogram was performed by Vitek 1 (from 2005 to 2006) and by Vitek 2 (from 2010 to 2011)
(bioMérieux, St. Louis, Missouri, USA). Card N104 was used in Vitek 2. Detection of extended spectrum beta-lactamases (ESBL) was performed on the samples from 2010 to 2011 by Vitek 2.

The standardization of the antibiogram followed the standards and recommendations of the Clinical and Laboratory Standards Institute (CLSI), documents M100-S15 (2005) ${ }^{5}$, M100-S16 (2006) ${ }^{6}, \mathrm{M} 100-\mathrm{S} 20$ $(2010)^{7}$ and M100-S21 (2011) ${ }^{8}$. Antibiograms of nonfermenting Gramnegative agents were confirmed by the E-test ${ }^{\circledR}$ (AB Biodisk, Solna, Sweden) and/or Kirby-Bauer disk diffusion ${ }^{18}$ methods, according to the same standards. No value of the minimum inhibitory concentration was computed in the analysis due to the lack of reliability of the automated antibiogram, especially the values obtained by Vitek 1. ATCC (American Type Culture Collection) strains of Escherichia coli (ATCC 25922), Klebsiella pneumoniae ESBL (ATCC 700063), Klebsiella pneumoniae (ATCC 700603), Pseudomonas aeruginosa (ATCC 27853) and Shigella sonnei (ATCC 25931) were used for quality control of Vitek analysis. For analysis purposes, we considered the frequency of susceptibility rather than resistance, which is different from most studies in the literature because the semiquantitative antibiogram also measures intermediate susceptibility. For the purposes of the analysis, intermediate susceptibility was included as resistance, as occurs in clinical practice.

A review of the literature was performed in MEDLINE with the terms "outpatient UTI" and "resistance to antibiotics" and "Brazil", as well as LILACS and SciELO (Scientific Electronic Library Online), which are Latin American scientific databases.

\section{ETHICS}

This study was approved by the Research Ethics Committee (Advisory Board) of the University Hospital of the University of São Paulo.

\section{STATISTICAL ANALYSIS}

Demographic data (sex, age and setting of the healthcare entrance at the hospital) and periods were submitted to exploratory data analysis and the assumption of normality. Numerical data were presented as the medians and interquartile ranges (IQR: Q1 and Q3) because the data were non-normal. Categorical data (sex, setting and susceptibility to antibiotics) were presented as the number and percentage. The MannWhitney U test was used for comparison of numerical non-normal variables. The Pearson uncorrected chi-square $\left(\chi^{2}\right)$ or Fisher's exact tests were used for comparison of susceptibility between the two periods (2005-2006 vs. 2010-2011). A crude (unadjusted or univariate) odds ratio, $95 \%$ confidence interval and $p$-value were used to assess risk factors for infection by the most frequently isolated bacteria. All tests were two-sided. Statistical significance was considered at $p<0.05$. Statistical analysis was performed using the statistical software SPSS 10.0 for Windows (IBM, Chicago, IL, USA).

\section{RESULTS}

In both periods analyzed, 11,943 aerobic urine cultures were obtained, $5,755(48.2 \%)$ from the period between 2005 and 2006 and 6,188 (51.8\%) from the period between 2010 and 2011. This is an average of 


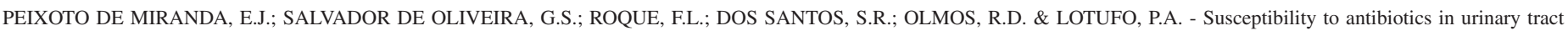
infections in a secondary care setting from 2005-2006 and 2010-2011, in São Paulo, Brazil: data from 11,943 urine cultures. Rev. Inst. Med. Trop. Sao Paulo, 56(4): 313-24, 2014.

7.88 positive urine cultures per day during the first period and 8.40 in the second. Only 849 (7.1\%) cultures presented counts less than 100,000 UFC in the first period (2005-2006).

The median age of patients was 36 years old (IQR: 20-65 years), ranging from zero to 100 years of age. There was a statistically significant difference between the median age of men and women in the study: 57.5 years old (IQR: 14.25-76 years) vs. 32 years old (IQR: 21-59 years), respectively; $p<0.0001$.

By age group, among patients 0 to 14 years old, the frequency of positive urine cultures was 2,007 (16.8\%); 15 to 64 years, 6,834 (57.2\%); and $\geq 65$ years, $3,102(26.0 \%)$. The female-to-male ratio per age strata was the following: $0.73(206 / 283)$ for $<2$ years old; $3.60(267 / 74)$ for $\geq 2$-5 years; 5.20 (229/44) for $>5$-14 years; 6.25 (2,794/447) for $\geq 14-64$ years; and $1.64(877 / 534)$ for $>65$ years $(p<0.0001$, Pearson chi-square).

The frequency of females was $77.6 \%$, with females between the ages of 14 and 64 representing $23.4 \%$ of the total. Among the patients studied, the majority of samples were collected in the Emergency Department, $8,931(74.8 \%)$. Other samples, $1,944(16.3 \%)$, were collected from the outpatient Clinic, and others, 1,059 (8.9\%), were considered "external" (patients from primary care units in the western region of São Paulo).

It was not possible to calculate the positivity rate of the urine cultures. Of all of the isolates, 10,891 (91\%) were Gram-negative, of which, 10,704 were Enterobacteriaceae (98.3\%) and $187(1.7 \%)$ were nonfermenting Gram-negative bacteria (manly Pseudomonas aeruginosa, 132; Acinetobacter baumannii, 36; and Stenotrophomonas maltophilia, 7). Seventy-five different bacterial species were isolated from the urine cultures (Table 1), but the most frequently observed species were Escherichia coli $(8,376,70.2 \%)$. The order of frequency of bacteria was statistically significant and varied between men and women $(p<0.0001)$ (Table 2).

The main risk factor for E. coli and Staphylococcus saprophyticus infection was female sex (Table 2) [crude odds ratio $(\mathrm{OR})=3.60,95 \%$ CI (confidence interval): 3.30 to $3.90 ; p<0.0001$ and $\mathrm{OR}=10.37,95 \%$ CI: $3.84-28.0 ; p<0.0001$, respectively]. Both bacteria were also more common in younger patients. E. coli was more common in patients less than 65 years old $[(\mathrm{OR})=1.98$ (95\% CI: 1.81 to 2.16$) ; p=0.033$, and $S$. saprophyticus was more prevalent in patients aged 14 to 65 years $[\mathrm{OR}=8.62$ (95\% CI: 4.77-15.58); $p<0.0001]$. For Proteus mirabilis, the main risk factor was male sex, even though an age of less than 65 years $(\mathrm{OR}=1.20,95 \% \mathrm{CI}: 1.01$ to $1.44 ; p=0.033)$ was also a risk factor. In the stratum of ages $0-14$, we observed the largest male-to-female ratio (1.7), whereas in the 15-64 year stratum it was 0.15 , and in the older age stratum ( $\geq 65$ years) it was 0.61 .

Male sex was also a risk factor for the following less frequent bacteria: Klebsiella pneumoniae, Enterococcus faecalis, Enterobacter aerogenes, . aeruginosa, Enterobacter cloacae, Citrobacter freundii and Morganella morganii (Table 2). The crosstabulation between stratum of age and gender resulted in a male-to-female rate in the age range of 65 years or greater divided per bacteria: 0.32 (E. coli), 1.023 (E. faecalis), 1.65 (E. cloacae), 1.625 (C. freundii), 3.25 (Staphylococcus epidermidis), 1.81 (Klebsiella oxytoca), 2.22 (E. aerogenes), 2.26 (S. aureus), and 1.875 (Citrobacter koseri).
Table 1

Frequency of species among 11,943 bacteria isolated from urine cultures collected between 2005-2006, and 2010- 2011 at the University Hospital of the University of São Paulo (HU-USP)

\begin{tabular}{llcc}
\hline Order & Bacteria isolated in urine culture & $\begin{array}{c}\text { Frequency } \\
(\mathrm{n})\end{array}$ & $\begin{array}{c}\text { Percentage } \\
(\%)\end{array}$ \\
\hline $1^{\circ}$ & Escherichia coli & 8,376 & $70.2 \%$ \\
$2^{\circ}$ & Proteus mirabilis & 795 & $6.8 \%$ \\
$3^{\circ}$ & Klebsiella pneumoniae & 692 & $5.9 \%$ \\
$4^{\circ}$ & Enterococcus faecalis & 322 & $2.8 \%$ \\
$5^{\circ}$ & Enterobacter aerogenes & 187 & $1.7 \%$ \\
$6^{\circ}$ & Staphylococcus saprophyticus & 148 & $1.2 \%$ \\
$7^{\circ}$ & Pseudomonas aeruginosa & 132 & $1.1 \%$ \\
$8^{\circ}$ & Enterobacter cloacae & 126 & $1.1 \%$ \\
$9^{\circ}$ & Citrobacter freundii & 122 & $1.0 \%$ \\
$10^{\circ}$ & Morganella morganii & 104 & $0.9 \%$ \\
$11^{\circ}$ & Staphylococcus aureus & 98 & $0.8 \%$ \\
$12^{\circ}$ & Staphylococcus epidermidis & 85 & $0.7 \%$ \\
$13^{\circ}$ & Citrobacter koseri & 82 & $0.7 \%$ \\
$14^{\circ}$ & Staphylococcus sp. DNAse & 65 & $0.5 \%$ \\
& negative & & \\
$15^{\circ}$ & Staphylococcus warneri & 62 & $0.5 \%$ \\
$16^{\circ}$ & Serratia marcescens & 62 & $0.5 \%$ \\
$17^{\circ}$ & Klebsiella oxytoca & 58 & $0.5 \%$ \\
$18^{\circ}$ & Streptococcus agalactiae & 43 & $0.4 \%$ \\
$19^{\circ}$ & Staphylococcus hominis & 38 & $0.3 \%$ \\
$20^{\circ}$ & Acinetobacter baumannii & 36 & $0.3 \%$ \\
$21^{\circ}$ & Others & 310 & $2.1 \%$ \\
\hline & Total & 11,943 & $100.0 \%$ \\
\hline
\end{tabular}

Notes: 1: neg.: negative, or Staphylococcus spp., neither classified as S. aureus (DNAse positive) nor had a specie discrimination, due to lack of discriminatory power by automated method (Vitek 1 or 2).

Appendix: Another isolates, in order of frequency, from the twenty-first: Staphylococcus haemolyticus, Enterococcus faecium, Streptococcus viridans, Streptococcus mitis, Proteus vulgaris, Staphylococcus simulans, Providencia stuartii, Providencia rettgeri, Staphylococcus sciuri, Salmonella spp., Stenotrophomonas maltophilia, Staphylococcus auricularis, Staphylococcus capitis, Citrobacter amalonaticus, Proteus penneri, Raoultella ornithinolytica, Raoultella planticola, Staphylococcus xylosus, Enterobacter asburiae, Enterococcus spp., Serratia fonticola, Serratia liquefaciens, Streptococcus anginosus, Streptococcus sanguinis, Aeromonas spp., Gram-negative (not discriminated), Burkholderia cepacia, Citrobacter braakii, Citrobacter spp., Enterobacter gergoviae, Enterobacter spp., Pseudomonas spp., coagulasenegative Staphylococcus spp., Staphylococcus intermedius, Staphylococcus lentus, Staphylococcus lugdunensis, Streptococcus bovis, Streptococcus spp. gallolyticus var. gallolyticus, Acinetobacter haemolyticus, Acinetobacter lwoffii, Acinetobacter spp., nonfermenting Gram-negative bacteria, Enterobacter sakazaki, Enterococcus hirae, Klebsiella ornithinolytica, Pantoea spp., Proteus spp., Providencia spp., Pseudomonas fluorescens, Pseudomonas putida, Staphylococcus cohnii spp. cohnii, Streptococcus gordonii, Streptococcus parasanguinis, Streptococcus pyogenes, beta-hemolytic group G Streptococcus spp.

Extended spectrum beta-lactamases (ESBL) were identified in 173 of the 5,722 Gram-negative isolates (3.0\%) that were collected between 2010 and 2011 and analyzed by the Vitek 2 . Of these, 108 were 
PEIXOTO DE MIRANDA, E.J.; SALVADOR DE OLIVEIRA, G.S.; ROQUE, F.L.; DOS SANTOS, S.R.; OLMOS, R.D. \& LOTUFO, P.A. - Susceptibility to antibiotics in urinary tract infections in a secondary care setting from 2005-2006 and 2010-2011, in São Paulo, Brazil: data from 11,943 urine cultures. Rev. Inst. Med. Trop. Sao Paulo, 56(4): 313-24, 2014.

Table 2

Frequency by gender of species among 11,943 bacteria isolated from urine cultures collected between 2005-2006, and 2010- 2011 at the University Hospital of the University of São Paulo (HU-USP)

\begin{tabular}{|c|c|c|c|c|c|}
\hline Order & Male, $n=2,673$ & Female, $\mathrm{n}=9,270$ & Odds ratio* & CI $95 \%$ & $p$ \\
\hline $1^{\circ}$ & Escherichia coli, 1,271 (47.5\%) & Escherichia coli, 7,103 (76.6\%) & $3.60(\mathrm{~F})$ & $3.30-3.90$ & $<0.0001$ \\
\hline $2^{\circ}$ & Proteus mirabilis, 302 (11.3\%). & Proteus mirabilis, 493 (5.31\%) & $2.27(\mathrm{M})$ & $1.94-2.64$ & $<0.0001$ \\
\hline $3^{\circ}$ & Klebsiella pneumoniae, 215 (8.04\%) & Klebsiella pneumoniae, 477 (5.1\%) & $1.61(\mathrm{M})$ & $1.36-1.91$ & $<0.0001$ \\
\hline $4^{\circ}$ & Enterococcus faecalis, 151 (5.65\%) & Enterococcus faecalis, 171 (1.8\%) & $3.19(\mathrm{M})$ & $2.53-4.01$ & $<0.0001$ \\
\hline $5^{\circ}$ & Morganella morganni, 77 (2.88\%) & Staphylococcus saprophyticus, 144 (1.5\%) & - & - & - \\
\hline $6^{\circ}$ & Pseudomonas aeruginosa, 76 (2.84\%) & Enterobacter aerogenes, 131 (1.4\%) & - & - & - \\
\hline $7^{\circ}$ & Enterobacter cloacae, 59 (2.20\%) & Enterobacter cloacae, $67(0.72 \%)$ & $3.09(\mathrm{M})$ & $2.17-4.41$ & $<0.0001$ \\
\hline $8^{\circ}$ & Citrobacter freundii, $56(2.10 \%)$ & Citrobacter freundii, $66(0.71 \%)$ & $2.98(\mathrm{M})$ & $2.08-4.27$ & $<0.0001$ \\
\hline $9^{\circ}$ & Enterobacter aerogenes, $56(2.10 \%)$ & Staphylococcus warneri, 59 (0.63\%) & - & - & - \\
\hline $10^{\circ}$ & Staphylococcus aureus, 52 (1.94\%) & Pseudomonas aeruginosa, $56(0.60 \%)$ & - & - & - \\
\hline
\end{tabular}

Notes: $*(\mathrm{~F})$ : female, reference is male; $(\mathrm{M})$ : male, reference is female (reference is the counterpart of the gender indicated in parentheses). Morganella morganni: $\mathrm{OR}=10.15$ (95\% CI: 6.53-15.76), $p<0.0001$ (male); Pseudomonas aeruginosa: $\mathrm{OR}=4.81$ (95\% CI: 3.40-6.82), $p<0.0001$ (male); Staphylococcus saprophyticus: $\mathrm{OR}=10.37$ (95\% CI: 3.84-28.0), $p<0.0001$ (female); Enterobacter aerogenes: $\mathrm{OR}=1.48$ (95\% CI: 1.08-2.02), $p=0.012$ (male).

Escherichia coli (1.3\% of total per specie), 59 were $K$. pneumoniae (8.5\%), four were K. oxytoca $(6.9 \%)$, and two were P. mirabilis $(0.25 \%)$. Of these, $4.6 \%(n=8)$ of patients were $0-14$ years old; $38.7 \%(n=67)$ were $15-64$ years; $56.7 \%(n=98)$ were $\geq 65$ years old; $60.1 \%(n=104)$ were female; and $74 \%(n=128)$ were from the Emergency Department. Of the patients $\geq 65$ years old, $55.1 \%$ were female and $44.9 \%$ were male.

Although it was neither carried out using the Hodge test nor another method for detecting carbapenemases, including KPC (K. pneumoniae carbapenemase), the susceptibility to ertapenem was high among isolates of Enterobacteriaceae (E. coli, 100\%; Proteus mirabilis, 99.5\%; K. pneumoniae, $98.5 \%$; and E. aerogenes, $100 \%$ ) during the period between 2010 and 2011. Global analysis of the isolates demonstrated a susceptibility of $99.8 \%$ to ertapenem (2010-2011). Susceptibility to vancomycin among isolates of E. faecalis was $98.6 \%$ and $97.3 \%$ ( $p=0.699)$, in the 2005-2006 and 2010-2011 periods, respectively.

\section{SUSCEPTIBILITY}

In an attempt to select an appropriate antibiotic for empirical treatment, we performed a comprehensive analysis of the antibiograms of 11,943 isolates from urine cultures from the two periods studied, despite differences in interpretation of susceptibility, particularly among Gram-positive and Gram-negative bacteria. Global susceptibility was $83.4 \%(67,864$ out of 81,406$)$, in $2005-2006$ vs. $83.5 \%(72,442$ out of 86,770 ) in 2010-2011, $p=0.499$. Only clindamycin, cefazolin, erythromycin, gentamicin (high level resistance, tested for Enterococcus spp. infection), linezolid, penicillin G (parenteral), tetracycline and vancomycin had no statistically significant differences in antimicrobial susceptibility in both periods. All of these antimicrobial agents are used for covering Gram-positive bacterial infections. All of the following antimicrobial agents were highly statistically significant different in the two periods $(p<0.0001)$ : nalidixic acid (88.7 vs. 78.1$)$, amikacin (98.4 vs. 99.5), amoxicillin/clavulanate (98.4 vs. 99.5), ampicillin/sulbactam (21.9 vs. 43.6), cephalothin (77.9 vs. 64.7), cefepime (98.0 vs. 95.4), ceftriaxone (98.2 vs. 95.9), ciprofloxacin (90.1 vs. 83.4), gentamicin (94.4 vs. 93.3), imipenem (99.8 vs. 93.1), meropenem ( 89.0 vs. 99.8), nitrofurantoin (86.4 vs. 77.4), norfloxacin (91.3 vs. 75.6) and piperacillin/ tazobactam (72.9 vs. 94.4). The difference was minor in the following cases: ampicillin (45.1 vs. 44.0, $p=0.037$ ), aztreonam 57 (48.3 vs. $61.5, p=0.048)$, cefuroxime axetil (92.1 vs. $86.9, p=0.004)$, oxacillin (22.4 vs. 35.6, $p=0.001)$, rifampin (96.4 vs. 97.7, $p=0.002)$ and trimethoprim/sulfamethoxazole (TMP/SMX) (68.0 vs. $65.9, p=0.035)$. Global resistance to fluoroquinolones (levofloxacin, norfloxacin and ciprofloxacin) was $12 \%$.

Among E. coli, the most commonly isolated agent, only TMP/SMX and nalidixic acid showed a resistance rate of $\geq 20 \%$, in the period from 2010 to 2011 . The global susceptibility was $88.3 \%(50,142 / 56,764)$ vs. $86.1 \%(55,436 / 64,400) ; p<0.0001$. Among $P$. mirabilis, except for colistin and nitrofurantoin, which were antibiotics against which this species has intrinsic resistance ( $0 \%$ susceptibility in this study) and TMP/ SMX (susceptibility of $77.8 \%$ and $74.5 \%$ in the first and second periods, respectively), all other antibiotics had $<20 \%$ resistance in both periods. K. pneumoniae had a global susceptibility of $80.8 \%(3,242 / 4,013)$ vs. $76.8 \%(4,719 / 6,150) ; p<0.0001$. Various antibiotics showed a resistance rate higher than $20 \%$, but norfloxacin was a good option in both periods that were studied $(89.7 \%$ vs. $82.6 \%)$. Among E. faecalis, the global susceptibility was $77.2 \%(880 / 1,141)$ vs. $82.2 \%(1,328 / 1,616) ; p=0.001$, which indicated an improvement in susceptibility during the two periods.

The most resistant species was E. aerogenes, with a global susceptibility of $70.9 \%(878 / 1,237)$ vs. $68.9 \%(1,012 / 1,468) ; p=0.674$. In contrast to the aforementioned bacteria, $S$. saprophyticus presented a favorable profile with an improvement in global susceptibility during the two periods, $61.1 \%(1,249 / 2,043)$ vs. $88.2 \%(15 / 17) ; p=0.042$. The reason that the number of samples that were obtained during the second period was small is because the laboratory is no longer disclosing the antibiogram for $S$. saprophyticus, as recommended by the CLSI. Among P. aeruginosa, the global susceptibility was $48.3 \%(532 / 1,102)$ in 2005 2006 vs. $85.6 \%(640 / 748)$ in $2010-2011 ; p<0.0001$.

The global frequency of intermediate susceptibility was the following: $1.7 \%$ (2005-2006) and 2.7\% (2010-2011) (global); $1.6 \%$ 


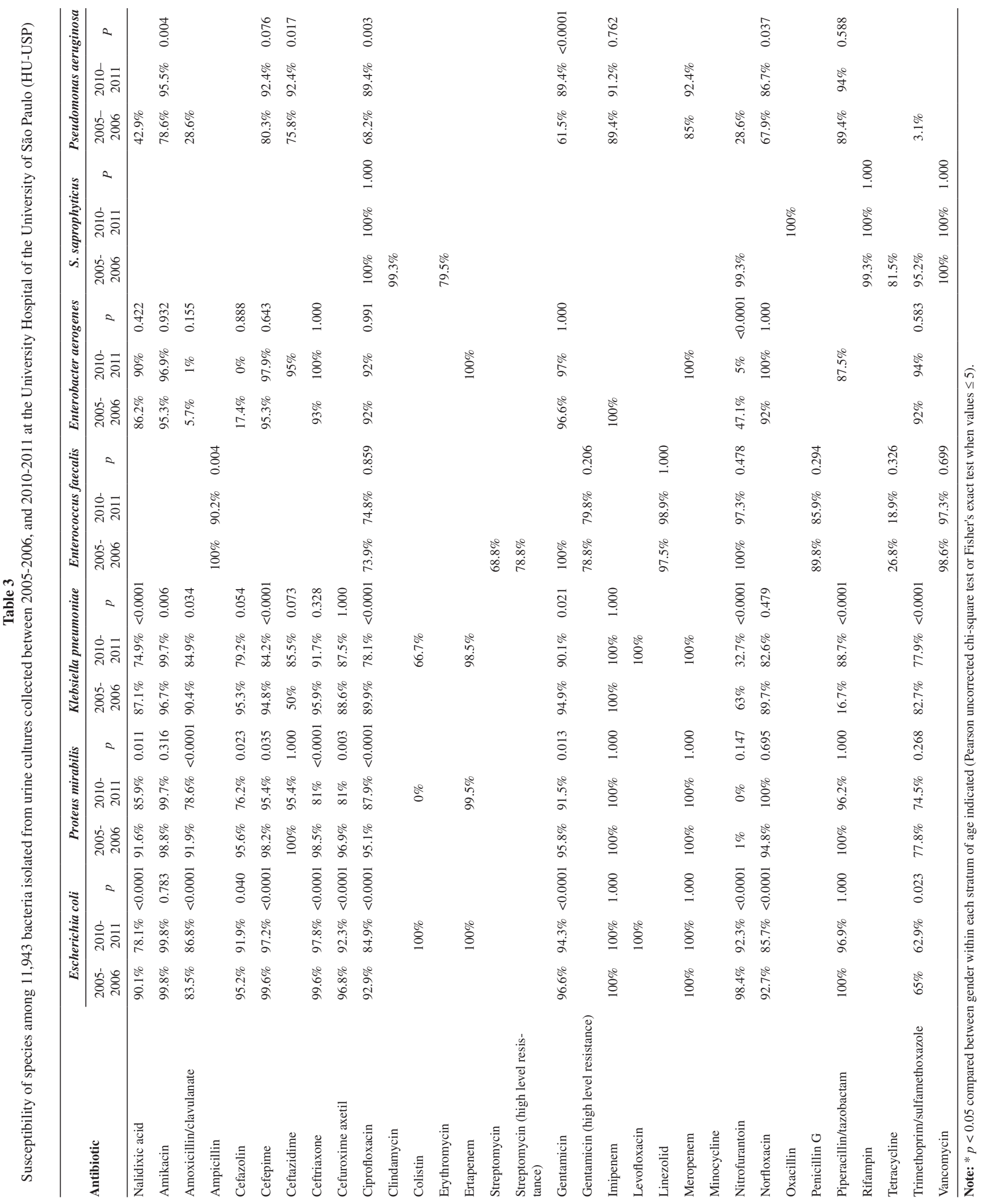




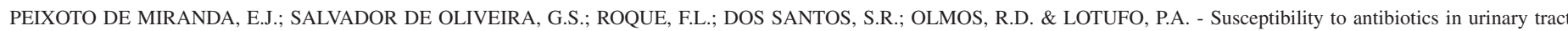
infections in a secondary care setting from 2005-2006 and 2010-2011, in São Paulo, Brazil: data from 11,943 urine cultures. Rev. Inst. Med. Trop. Sao Paulo, 56(4): 313-24, 2014.

and $2.7 \%$ (E. coli); $0.8 \%$ and $2.5 \%$ (Proteus mirabilis); $3.0 \%$ and $3.5 \%$ (K. pneumoniae); $0.5 \%$ and $2.0 \%$ (E. faecalis); $3.6 \%$ and $3.7 \%$ (E. aerogenes); $0.5 \%$ and $0 \%$ (S. saprophyticus); and $4.6 \%$ and $5.3 \%$ ( $P$. aeruginosa).

The comparison among the age strata, 0-14, 15-64 and $\geq 65$ years old, versus gender, among the 45 antibiotics that were tested (Table 4), showed statistically significant differences for 25 of the antibiotics between males and females, with greater rates of resistance in men and in patients older than 65 years. The antibiotics demonstrating these differences were the following: nalidixic acid, amikacin, amoxicillin/ clavulanate, ampicillin, ampicillin/sulbactam, aztreonam, cephalothin, cefazolin, cefepime, cefotaxime, ceftazidime, ceftriaxone, cefuroxime axetil, ciprofloxacin, carbenicillin, ertapenem, streptomycin (high level resistance), gentamicin, imipenem, meropenem, nitrofurantoin, piperacillin/tazobactam, ticarcillin, TMP/SMX and vancomycin.

\section{DISCUSSION}

This is the third largest study of susceptibility to antibiotics in urinary tract infections in Brazil (Table 5). All studies of this type in Brazil, except for one ${ }^{10}$, are retrospective and exclude many demographic variables, except gender and age, as well as clinical, radiological or laboratory variables, which does not allow multivariate analysis, and thus, greatly reduces the impact of these publications ${ }^{1,2,4,9,19,20,25,26}$.

Initially, this study had the goal of guiding the implementation of a protocol for the treatment of UTIs; however, the lack of clinical data (mainly regarding the uncomplicated infections) and the large number of isolates, preclude such an achievement.

Considering that $\$ 5.30$ US dollars was paid for each urine culture and antibiogram according to the table of Brazilian Unified Health System, the "estimated cost" of this study was $\$ 63,297.90^{27}$. Taking into account that in the same period approximately 60,000 urine cultures were requested, of which, only $20 \%$ were positive, the total cost to the health system was about $\$ 183,000.00$ just in the University Hospital of the University of São Paulo, which is one of 6,753 hospitals in Brazil, of which only $30 \%$ are public. This justifies conducting a prospective study to address the clinical characteristics of patients in Brazil.

The biggest (multicenter) impact study involving Brazilian cases of UTI isolates was the SENTRY ${ }^{13}$, which was published a decade ago and involved four centers in the country but only included inpatients. The methodology included the use of the gold standard procedure of microdilution for the preparation of the antibiograms and involved 1,961 urine isolates. E. coli was the most frequent pathogen that was isolated followed by Klebsiella spp., P. aeruginosa, and P. mirabilis. At that time, the amount of detected resistance was $>45 \%$ to TMP/ SMX and a resistance to fluoroquinolones of 17.5-18.9\% was detected. Furthermore, the susceptibility to broad-spectrum antibiotics (91.0$100.0 \%$ ) and nitrofurantoin (87\%) remained high, including piperacillin/ tazobactam, aztreonam, extended-spectrum cephalosporins (third and fourth generations), carbapenems and amikacin. At that time, the biggest problems in terms of resistance was carbapenem-resistant $P$. aeruginosa, ciprofloxacin-resistant $E$. coli and ESBL-producing $K$. pneumoniae. The Enterobacteriaecae producing carbapenemases were not even mentioned in that study ${ }^{13}$.
The data found in this study are similar to those found in previous literature, with some minor peculiarities. The proportion of females ( $77.6 \%$ vs. $69 \%-88.8 \%$ in studies), the female-to-male ratio by age group, the age group most affected (15-64 years: 57.2\%) and the first isolated agent $($ E. coli, $70.2 \%)$ and frequency is very similar to that found in many published studies $2,4,9,10,15,19,20,25,26$. The percentage of $E$. coli that is isolated from UTIs, according to international literature, is $75 \%$ to $95 \%{ }^{15}$. In Brazil, the frequency varied from $48.2 \%{ }^{9}$ to $71.6 \%^{19}$ in São Paulo. The proportion of Gram-negative isolates varied from $84.4 \%$ to $87.3 \%{ }^{4,19,24}$.

The second most frequent agent presented a variation in different studies in Brazil. Except in studies by COSTA et al. ${ }^{9}$ and $\mathrm{KOCH}$ et al. ${ }^{20}$, in which Proteus mirabilis was the second most frequently isolated uropathogen, the second most commonly isolated pathogen was Enterococcus spp. ${ }^{25}$, K. oxytoca and $S$. agalactiae ${ }^{22}$, Enterobacter spp. ${ }^{2}$, and $K$. pneumoniae pr,11,19,22. $^{2}$.

In the one Brazilian study to date ${ }^{11}$ about ESBL-producing Enterobacteriaceae in community-acquired UTIs, which evaluated 78,964 urine cultures in Juiz de Fora, State of Minas Gerais, Southeastern of Brazil, from 2001 to 2009, detected a frequency of $0.66 \%(n=512)$ over nine years in the majority of patients older than 65 years (female, $52.6 \%$ and male, $48.1 \%$ ), which is similarly to the results of this study. The percentage reported in the literature varied from $0.2 \%$ to $3.5 \%$, which is close to the value of $3.0 \%$ among Gram-negative tested between 2010 and 2011, in this study. Although the most frequently isolated bacterium that produces ESBL is Klebsiella spp. (24 to 60\%), DIAS et al. also detected E. coli (72.4\%), followed by Klebsiella spp. (19.8\%), which is similar to this study in which E. coli $(62.4 \%)$, Klebsiella spp. (36.4\%) and Proteus spp. (1.2\%) were isolated. This study did not perform a confirmatory test for ESBL, which can result in false positives ${ }^{12}$.

Global resistance was $\geq 20 \%$ for TMP/SMX, tetracycline, norfloxacin, nitrofurantoin, erythromycin, cefazolin and nalidixic acid, especially leveraged by other bacteria than E. coli. Global resistance was $\geq 20 \%$ for TMP/SMX, tetracycline, norfloxacin, nitrofurantoin, erythromycin, cefazolin and nalidixic acid in the isolated bacteria other than E. coli. There was significant difference between virtually all antibiotics in the two periods studied, which was commonly highly significant $(p<0.0001)$; however, no difference was observed between the antibiotics used for coverage of Gram-positive bacteria, except for rifampin and oxacillin. The fact that there was no statistically significant difference between the two periods in the overall susceptibility to key antibiotics by Gram-positive bacteria reveals the minor importance of these bacteria in the pathogenesis of UTIs in the study population, due to the reduced presence of selective pressure, which occurs with antibiotics that are used to treat Gram-negative bacteria.

The study by $\mathrm{KOCH}$ et $a l .{ }^{20}$ assessed the temporal trends of antimicrobial resistance, from 2000 to 2004 of gentamicin $(p=0.98)$, cephalothin $(p=0.29)$, cefoxitin $(p=0.16)$, TMP/SMX $(p=0.40)$, nalidixic acid $(p=0.02)$, ceftriaxone $(p=0.12)$ and ciprofloxacin $(p=0.07)$. Therefore, unlike this study, only nalidixic acid had statistical significance and ciprofloxacin had a statistical trend, which demonstrates the selective pressure that was exerted by quinolones in Rio Grande do Sul, southern Brazil. The same study also showed that older patients showed increased resistance to nalidixic acid, ciprofloxacin and 


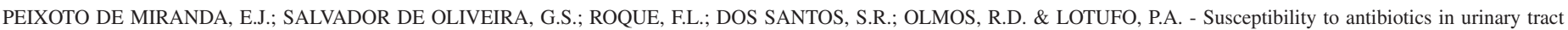
infections in a secondary care setting from 2005-2006 and 2010-2011, in São Paulo, Brazil: data from 11,943 urine cultures. Rev. Inst. Med. Trop. Sao Paulo, 56(4): 313-24, 2014.

Table 4

Susceptibility to antibiotics of all 11,943 bacteria isolated from urine cultures, between 2005 and 2006 , and 2010 and 2011 at the University Hospital of the University of São Paulo (HU-USP), stratified by gender and stratum of age

\begin{tabular}{|c|c|c|c|c|c|c|}
\hline \multirow{2}{*}{ Antibiotic, susceptible, $\mathrm{n}(\%)$} & \multicolumn{3}{|c|}{ Male gender } & \multicolumn{3}{|c|}{ Female gender } \\
\hline & $0-14$ years & 15-64 years & $\geq 65$ years & $0-14$ years & 15-64 years & $\geq 65$ years \\
\hline Nalidixic acid & $603(97.3)^{*}$ & $532(74.2)^{*}$ & $506(59.3)^{*}$ & $1.198(94.1)^{*}$ & $4.794(88.1)^{*}$ & $1.220(69.8)^{*}$ \\
\hline Amikacin & $528(100)$ & $635(96.1)^{*}$ & $794(95.0)^{*}$ & $1.107(100)$ & $4.740(99.8)^{*}$ & $1.569(99.2)^{*}$ \\
\hline Amoxicillin/clavulanate & $454(73.2)^{*}$ & $490(68.3)^{*}$ & $530(62.1)^{*}$ & $1.050(82.5)^{*}$ & $4.637(85.2)^{*}$ & $1.395(79.8)^{*}$ \\
\hline Ampicillin & $253(38.8)$ & $304(37.7)^{*}$ & $318(31.3)^{*}$ & $539(41.2)$ & $2.802(48.6)^{*}$ & $880(46.5)^{*}$ \\
\hline Ampicillin/sulbactam & $3(16.7)$ & $17(29.8)^{*}$ & $40(44.9)^{*}$ & $2(11.1)$ & $39(17.1)^{*}$ & $14(23.3)^{*}$ \\
\hline Aztreonam & $9(100)$ & $25(49.0)$ & $31(41.9)^{*}$ & $18(85.7)$ & $11(45.8)$ & $27(64.3)^{*}$ \\
\hline Cephalothin & $457(73.2)^{*}$ & $448(60.3)^{*}$ & $443(49.4)^{*}$ & $964(75.4)^{*}$ & $4.154(76.2)^{*}$ & $1.185(66.9)^{*}$ \\
\hline Cefazolin & $87(77.0)^{*}$ & $102(68.5)^{*}$ & $106(70.7)^{*}$ & $178(87.7)^{*}$ & $741(73.0)^{*}$ & $217(83.8)^{*}$ \\
\hline Cefepime & $527(99.8)$ & $604(91.4)^{*}$ & $725(86.5)^{*}$ & $1.095(98.9)^{*}$ & $4.692(98.7)^{*}$ & $1.499(94.8)^{*}$ \\
\hline Cefotaxime & $230(95.0)$ & $296(81.8)^{*}$ & $374(73.3)^{*}$ & $549(95.8)$ & $2.717(97.2)^{*}$ & $854(88.5)^{*}$ \\
\hline Cefoxitin & 0 & $4(14.8)^{*}$ & $3(6.8)$ & $5(45.5)$ & $17(65.4)^{*}$ & $4(16.0)$ \\
\hline Ceftazidime & $254(98.8)$ & $349(87.5)^{*}$ & $444(81.0)^{*}$ & 609 (97.9) & $2.902(97.7)^{*}$ & $1.383(87.7)^{*}$ \\
\hline Ceftriaxone & $383(98.7)^{*}$ & $374(91.9)^{*}$ & $382(91.0)^{*}$ & $705(100)^{*}$ & $2.677(99.5)^{*}$ & $807(97.6)^{*}$ \\
\hline Cefuroxime axetil & $272(93.2)^{*}$ & $243(77.9)^{*}$ & $227(70.7)^{*}$ & $528(97.8)^{*}$ & $1.954(96.1)^{*}$ & $557(88.8)^{*}$ \\
\hline Ciprofloxacin & $657(98.8)$ & $641(76.2)^{*}$ & $692(63.0)^{*}$ & $1.308(98.2)$ & $5.519(92.5)^{*}$ & $1.446(74.7)^{*}$ \\
\hline Clindamycin & $12(100)$ & $21(63.6)^{*}$ & $41(75.9)$ & $14(100)$ & $263(98.9)^{*}$ & $25(58.1)$ \\
\hline Chloramphenicol & - & - & $1(100)$ & - & $2(100)$ & - \\
\hline Carbenicillin & $32(37.6)$ & $52(61.9)$ & $32(45.7)^{*}$ & $59(39.6)$ & $306(55.8)$ & $97(60.2)^{*}$ \\
\hline Colistin & - & $3(75.0)$ & $2(100)$ & $1(100)$ & $4(80.0)$ & $4(100)$ \\
\hline Erythromycin & $9(75.0)$ & $16(48.5)^{*}$ & $31(57.4)$ & $13(86.7)$ & $216(81.2)^{*}$ & $23(53.5)$ \\
\hline Ertapenem & $231(100)$ & $318(99.4)$ & $446(98.9)^{*}$ & $563(100)$ & 2778 (99.9) & $933(99.9)^{*}$ \\
\hline Streptomycin & $7(58.3)$ & $10(62.5)$ & $38(73.1)$ & $5(55.6)$ & $14(63.6)$ & $30(62.5)$ \\
\hline Streptomycin (high level resistance) & $10(83.3)$ & $15(57.7)$ & $39(76.5)^{*}$ & $8(100)$ & $35(72.9)$ & $18(34.0)^{*}$ \\
\hline Gentamicin & $623(97.0)$ & $702(86.8)^{*}$ & $855(84.5)^{*}$ & $1.271(96.6)$ & $5.617(96.4)^{*}$ & $1.680(91.0)^{*}$ \\
\hline Gentamicin (high level resistance) & $21(87.5)$ & $27(64.3)^{*}$ & $80(77.7)$ & $17(100)$ & $64(91.4)^{*}$ & $70(70.0)$ \\
\hline Imipenem & $296(100)$ & $338(98.5)^{*}$ & $383(98.0)^{*}$ & $545(100)$ & $1.975(100)^{*}$ & $646(99.5)^{*}$ \\
\hline Levofloxacin & - & - & - & - & $7(100)$ & $3(100)$ \\
\hline Linezolid & $24(100)$ & $55(100)$ & $117(99.2)$ & $22(100)$ & $205(98.6)$ & $96(99.0)$ \\
\hline Meropenem & $241(100)$ & $362(98.4)^{*}$ & $509(97.7)^{*}$ & $582(100)$ & $2.800(100)^{*}$ & $968(99.7)^{*}$ \\
\hline Minocycline & $50(58.8)^{*}$ & $58(69.0)$ & $47(67.1)$ & $115(77.2)^{*}$ & $393(71.7)$ & $115(71.4)$ \\
\hline Nitrofurantoin & $389(60.2)^{*}$ & $584(74.8)^{*}$ & $660(67.7)^{*}$ & $1.119(86.2)^{*}$ & $5.010(86.8)^{*}$ & $1.515(81.7)^{*}$ \\
\hline Norfloxacin & $400(98.0)$ & $363(80.6)^{*}$ & $338(65.6)^{*}$ & $733(98.4)$ & $2.664(94.7)^{*}$ & $712(79.3)^{*}$ \\
\hline Oxacillin & $5(29.4)$ & $16(39.0)^{*}$ & $43(51.8)$ & $7(31.8)$ & $67(18.6)^{*}$ & $21(37.5)$ \\
\hline Penicillin $\mathrm{G}$ & $23(54.8)$ & $38(44.2)^{*}$ & $89(47.1)$ & $19(49.6)$ & $112(23.9)^{*}$ & $95(57.6)$ \\
\hline Piperacillin/tazobactam & $131(97.8)$ & $184(84.4)^{*}$ & $258(81.9)^{*}$ & $306(96.5)$ & $1.326(96.4)^{*}$ & $503(93.8) *$ \\
\hline Polymyxin B & $2(100)$ & $15(100)$ & $23(100)$ & $13(100)$ & $4(100)$ & $11(100)$ \\
\hline Quinupristin/dalfopristin & $3(50.0)$ & $11(68.8)$ & $17(56.7)$ & $1(33.3)$ & $28(93.3)$ & $10(45.5)$ \\
\hline Rifampin & $17(100)$ & $35(85.4)^{*}$ & $78(94.0)$ & $22(100)$ & $361(99.7)^{*}$ & $51(89.5)$ \\
\hline Teicoplanin & $16(100)$ & $27(100)$ & $59(89.4)$ & $17(100)$ & $135(99.3)$ & $61(98.4)$ \\
\hline Tetracycline & $20(47.6)^{*}$ & $50(54.9)^{*}$ & $81(54.4)$ & $45(71.4)^{*}$ & $350(66.5)^{*}$ & $78(48.4)$ \\
\hline Ticarcillin & $73(81.1)^{*}$ & $86(78.9)^{*}$ & $82(73.2)^{*}$ & $141(91.0)^{*}$ & $520(93.4)^{*}$ & $162(89.5)^{*}$ \\
\hline Tigecycline & - & $3(75.0)$ & $2(100)$ & $1(100)$ & $4(80.0)$ & $3(100)$ \\
\hline Tobramycin & $85(100)$ & $83(98.8)$ & $71(98.6)$ & $147(98.7)$ & $547(99.5)$ & $159(98.8)$ \\
\hline Trimethoprim/sulfamethoxazole & $415(65.5)$ & $485(63.0)^{*}$ & $564(59.2)^{*}$ & $807(62.4)$ & $4.007(70.6)^{*}$ & $1.176(65.1)^{*}$ \\
\hline Vancomycin & $43(100)$ & $93(98.9)$ & $190(94.1)^{*}$ & $43(100)$ & $496(100)$ & $170(98.8)^{*}$ \\
\hline
\end{tabular}

Note: $* p<0.05$ compared between gender within each stratum of age indicated (Pearson uncorrected chi-square test or Fisher's exact test when values $\leq 5)$. 


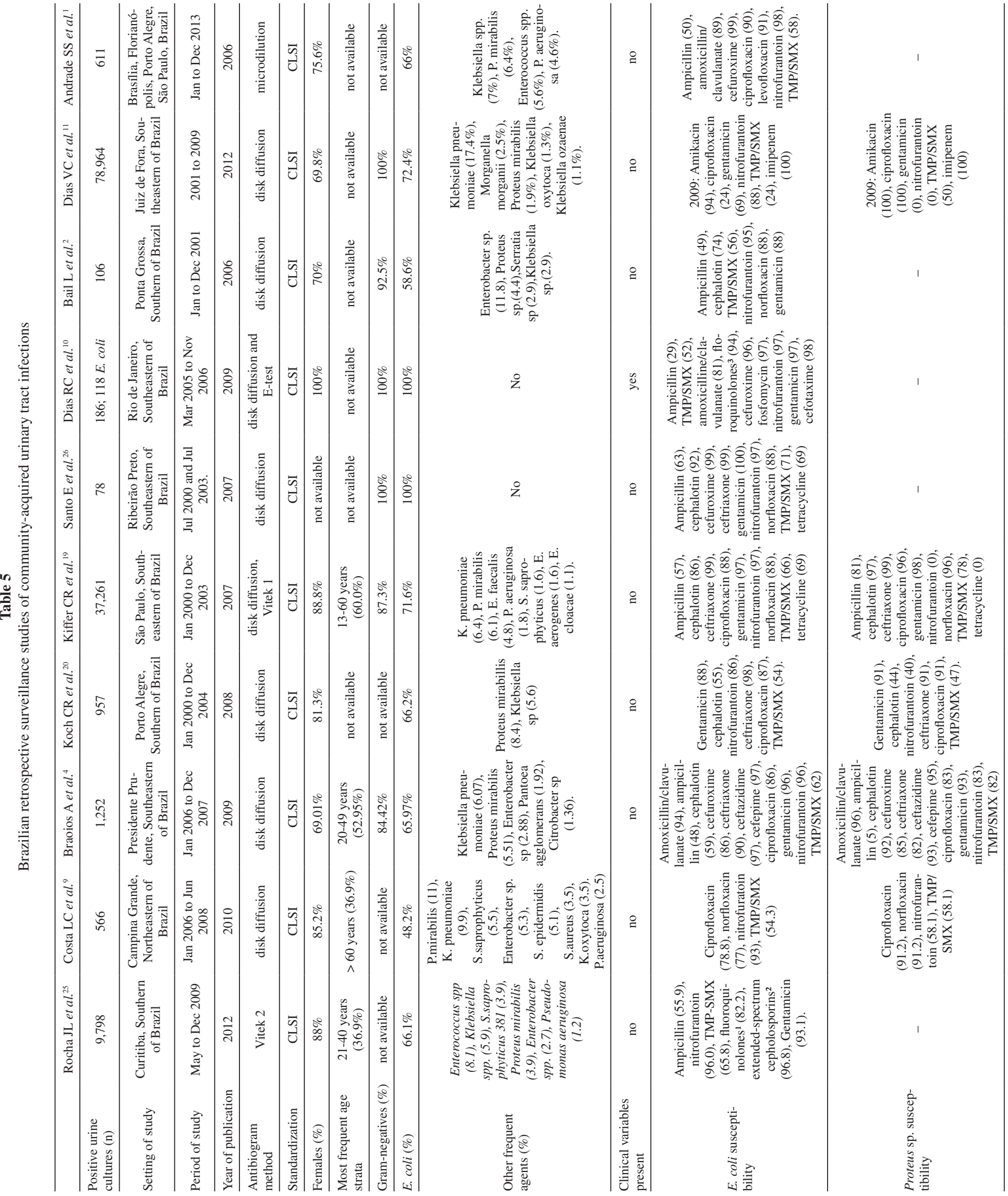




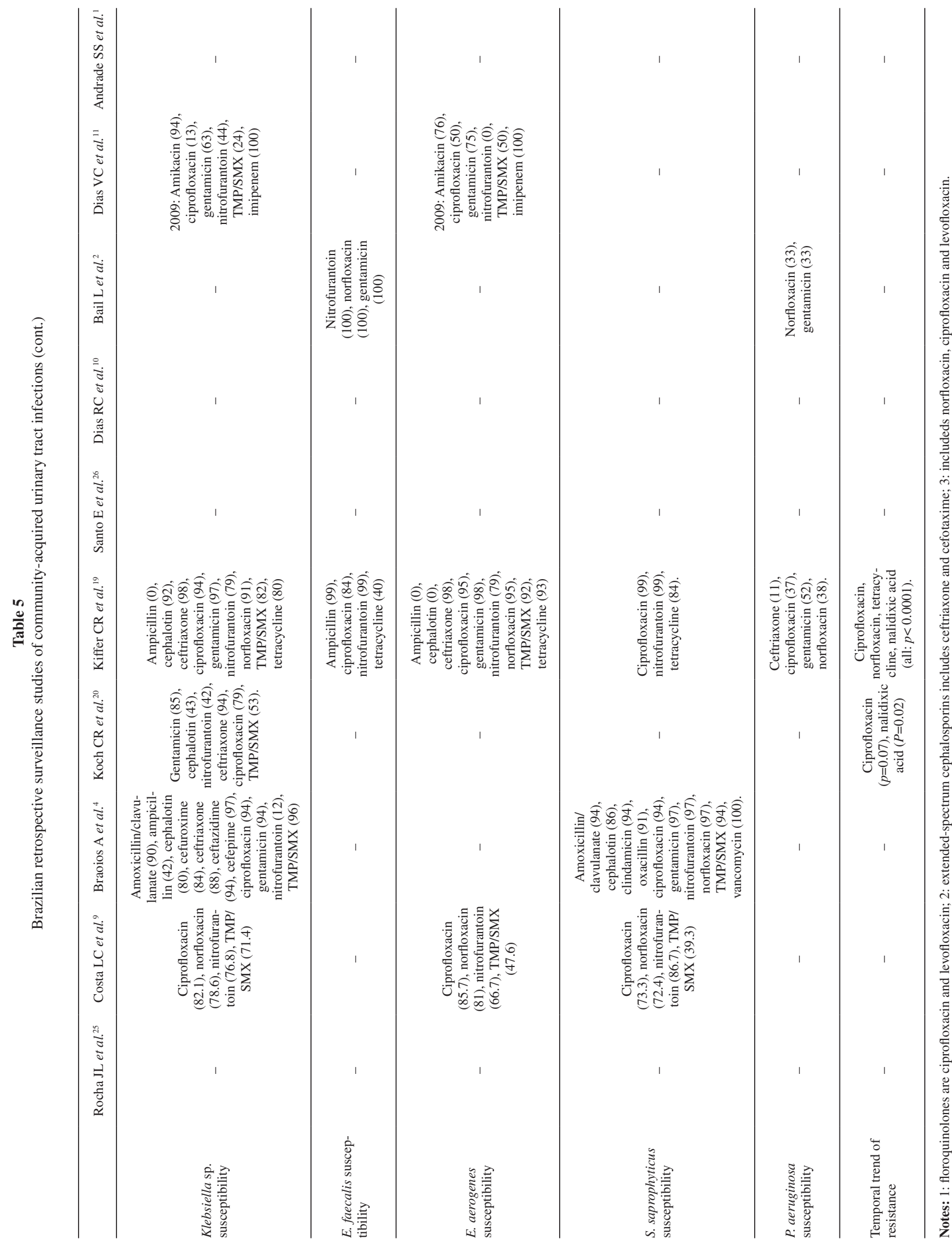




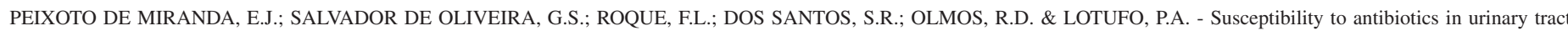
infections in a secondary care setting from 2005-2006 and 2010-2011, in São Paulo, Brazil: data from 11,943 urine cultures. Rev. Inst. Med. Trop. Sao Paulo, 56(4): 313-24, 2014.

nitrofurantoin, and in men, for many of the antibiotics that were tested, with the exception of TMP/SMX ${ }^{20}$. KIFFER et al. ${ }^{19}$ demonstrated a temporal trend of a decreased susceptibility to quinolones from 2000 to 2003 including ciprofloxacin, norfloxacin, tetracycline, and nalidixic acid $(p<0.0001)$. An earlier study, that was conducted two decades ago in Salvador, Northeastern Brazil, showed a reported resistance to norfloxacin that reached $9.1 \%$ and to ciprofloxacin of $16.5 \%{ }^{21}$.

The study by DIAS et al. ${ }^{11}$ evaluated the temporal trend of resistance among ESBL-producing bacteria and showed a peak of occurrence in 2005 (2000-2009) for all antibiotics close to 80\% (amikacin, ciprofloxacin, gentamicin, nitrofurantoin, TMP/SMX and imipenem) and a second peak in 2008 (ciprofloxacin and TMP/SMX were close to $80 \%$ and nitrofurantoin was close to $40 \%$ ).

The susceptibility of E. coli in 10 Brazilian studies of communityacquired UTIs showed that only TMP/SMX, cephalothin and norfloxacin presented $<80 \%$ susceptibility ${ }^{1,2,4,9,10,11,19,20,25,26}$. In addition to nitrofurantoin, to which $P$. mirabilis presents intrinsic resistance, TMP/ SMX and cephalothin were not good options for treatment of UTIs. According to other studies in Brazil, K. pneumoniae retains relative susceptibility to fluoroquinolones, as shown in this study, and only TMP/SMX and nitrofurantoin were not good options for treatment of UTIs. The profile found in the literature for E. aerogenes is similar to that found in our study, although the literature data indicate a decreased susceptibility to TMP/SMX ${ }^{9,11,19}$.

Except for lower levels of susceptibility to tetracycline and ciprofloxacin, the data regarding E. faecalis from this study resembles that found in the study by KIFFER et al. ${ }^{19}$. Data regarding $S$. saprophyticus demonstrated distinct profiles of susceptibility, and are similar to the data found in this study. Finally, there is a notable discrepancy between the data regarding the susceptibility of $P$. aeruginosa in this study and that of KIFFER et al. ${ }^{19}$, the latter being characterized by bacteria with more resistance.

A preliminary conclusion of the risk factors could indicate a predominance of $E$. coli in young women and a predominance of other more resistant isolates, particularly of Proteus spp. in men, which, according to literature, are most affected by urinary infections when they develop benign prostatic hyperplasia (BPH), even though the odds ratio is statistically significant for patients younger than $65^{14,15}$. Data from a higher incidence of resistance in elderly men are corroborated by three other Brazilian studies ${ }^{19,20,25}$. ROCHA et al. ${ }^{25}$ also demonstrate a statistically significant difference of the frequency of bacteria between men and women. They found a predominance of Enterococcus spp., Enterobacter spp. and Pseudomonas spp., but not Proteus spp. in men and a predominance of $S$. saprophyticus and E. coli in women. The same study also demonstrated a highly statistically significant susceptibility to antibiotics by E. coli and others, which corroborates the data from this study.

The favorable resistance profile of $E$. coli results in an expanded list of oral therapeutic options for cystitis treatment in young women, including pregnant women, such as fluoroquinolones for not pregnant patients, and cephalexin, cefuroxime axetil and amoxicillin/clavulanate for pregnant patients. However, a policy of sparing fluoroquinolones in such cases is urgently needed. Although it was previously a good option for young women, nitrofurantoin had low levels of susceptibility by the bacteria that were frequently isolated, such as Proteus spp. (intrinsically resistant), Klebsiella spp., Enterobacter spp. and Pseudomonas spp., as well as $M$. morganii and $S$. marcescens (both intrinsically resistant).

Therefore, the biggest problem with making recommendations for empiric antibiotics according to this casuist is men and the elderly. P. mirabilis, a urease-producing bacteria that is capable of causing struvite urolithiasis and was the second most frequent isolate, presents intrinsic resistance to several antibiotics, such as cefazolin, tigecycline, polymyxins and nitrofurantoin. Therefore, any empirical recommendation for men and elderly people, cannot dispense with fluoroquinolones or a reassessment based on early urine cultures.

For complicated infections and pyelonephritis, fluoroquinolones, which have renal penetration, such as ciprofloxacin, moxifloxacin and levofloxacin (although the latter two are reserved for respiratory infections because of their expanded spectrum for Gram-positive bacteria and atypical bacteria) are also good choices, although third and fourth generation cephalosporins, as well as aminoglycosides, are a second option and are preferred in cases of severe sepsis and septic shock. Amoxicillin/clavulanate is a good oral treatment option, especially after a loading dose of extended-spectrum cephalosporins or aminoglycosides.

Fosfomycin, although not evaluated by the antibiograms in this study, had a good susceptibility profile in a small Brazilian study $(97.0 \%$ of susceptibility, $\mathrm{n}=118)^{10}$ and is an option for multidrug-resistant infections, especially for ESBL- and carbapenemase-producing bacteria.

An elegant study from the viewpoint of mathematical modeling in the city of São Paulo, Brazil, by KIFFER et al. ${ }^{18}$ in 2002, demonstrated the correlation between the probability of resistance of strains of $E$. coli in urine and the "density the ciprofloxacin usage for the previous three months" (defined as D_DDDA), $p=0.023$. Additionally, ciprofloxacin D_DDDA was found to be related to clusters $^{18}$ : the western region of São Paulo was deemed a hot spot for the use of ciprofloxacin (average probability of 0.8808 ). This study is an elegant demonstration of how the indiscriminate use of ciprofloxacin against uropathogens may result in the loss of a medication to treat a broad spectrum of uropathogens. Furthermore, taking into account that approximately $30 \%$ to $45 \%$ of ciprofloxacin ingested is excreted in a non-metabolized form in the urine, widespread use can have environmental consequences due to presence of the antibiotic in the sewage and exposure of Enterobacteriaceae to the antibiotic in that environment. This finding only reinforces the urgent need, based on studies, for an efficient policy of reducing the use of fluoroquinolones and broader-spectrum antibiotics.

A recent study in the United States ${ }^{17}$ showed a higher risk of levofloxacin-resistant $E$. coli related to previous use of this antibiotic (adjusted $\mathrm{OR}=7.6,9 \% \mathrm{CI}: 2.1-27.5, p=0.002$ ) and previous hospitalization $(\mathrm{OR}=2.0,95 \% \mathrm{CI}: 1.0-3.9, p=0.04)$ as well as a statistically significant risk to resistance to other antibiotics in isolates resistant to this fluoroquinolone. Another interesting finding was increased rates of resistance to TMP/SMX despite a 50\% decrease in the selective pressure, which is most likely due to multidrug resistance plasmids or multidrug efflux pumps.

This study has several limitations, even though it has the merit of 


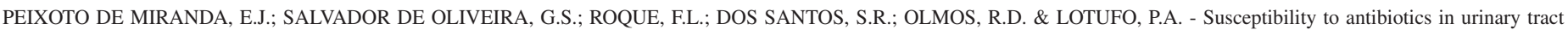
infections in a secondary care setting from 2005-2006 and 2010-2011, in São Paulo, Brazil: data from 11,943 urine cultures. Rev. Inst. Med. Trop. Sao Paulo, 56(4): 313-24, 2014.

being one of the few and largest of its type in Brazil: there is one small study of the antimicrobial susceptibility profile according to clinical presentation. In our analysis, we do not separate nosocomial infections from those acquired in the community. The samples were collected not only from midstream but also by other techniques, such as indwelling catheter, which can increase the chance of isolating hospital germs. Despite this, our resistance rates are similar to studies that included only community isolates. Our analysis was based on retrospective data and focused on microbiological aspects. Reproducibility problems can arise due to differences in interpretation of the minimum inhibitory concentration (MIC) between CLSI and EUCAST (European Committee on Antimicrobial Susceptibility Testing) documents.

Studies assessing other demographic, clinical, radiological and laboratory risk factors (including multivariate analysis to control for confounding factors) for infections by germs other than E. coli, such as Proteus spp., Enterobacter spp., Pseudomonas spp. and Klebsiella spp., are urgently needed, to make treatment recommendations that can save broad-spectrum drugs, such as fluoroquinolones, and third and fourth generations of cephalosporins, carbapenems, fosfomycin and aminoglycosides.

\section{CONCLUSIONS}

The most favorable scenario is the treatment of $E$. coli. TMP/SMX and nalidixic acid were only two whose resistance exceeds the threshold of $20 \%$, which contraindicates their use according to the guidelines, in our study. The rise of resistance to fluoroquinolones is concerning and averaged $12 \%$. Furthermore, the $20 \%$ threshold resistance was exceeded by norfloxacin in the overall analysis, mainly at the expense of bacteria other than E. coli. In this study, a high frequency of community-acquired ESBL bacteria was found, which is greater than that found in a study in Brazil, but within the range described in the literature. Most isolates were observed in the elderly. As in other studies, the main problem of resistance was noted in men and in the elderly, which limits most conservative treatment recommendations that save broad-spectrum antibiotics such as fluoroquinolones, aminoglycosides and third and fourth generations cephalosporins. Well-controlled prospective studies that evaluate the risk factors for UTIs by bacteria other than E. coli and resistance in Brazil are greatly needed.

\section{RESUMO}

\section{Suscetibilidade a antibióticos em infecções de trato urinário em um hospital secundário, 2005-2006 e 2010-2011, em São Paulo, Brasil: dados de 11.943 uroculturas}

Introdução: A infecção do trato urinário (ITU) tem alta incidência e recorrência, e o tratamento é empírico na maioria dos casos. Objetivos: O objetivo deste estudo foi analisar as culturas de urina realizadas em um hospital secundário, durante dois períodos: 2005-2006 e 2010-2011, para estimar a resistência microbiana. Pacientes e métodos: Foram analisadas 11.943 culturas aeróbicas de urina de acordo com um conjunto de dados demográficos básicos e susceptibilidade aos antibióticos, obedecendo às normas do Clinical and Laboratory Standards Institute (CLSI) para Vitek 1 e 2. Resultados: A maioria dos participantes era adulta e jovem atendida no Serviço de Emergência. E. coli foi a mais freqüente $(70,2 \%)$ entre as 75 espécies isoladas. Resistência de todos os isolados foi $\geq 20 \%$ para sulfametoxazol/trimetoprim (SMX/TMP), norfloxacina, nitrofurantoína, cefazolina e ácido nalidíxico, apesar de $E$. coli ter sido mais suscetível (resistência $\geq 20 \%$ apenas para SMX/TMP e ácido nalidíxico) entre todos os isolados, levando em conta a porcentagem de resistência e o número de antibióticos testados. Resistência às fluoroquinolonas foi de $12 \%$. Fatores de risco para E. coli: sexo feminino e idade $<65$ anos. Homens e pacientes com mais de 65 anos apresentaram isolados mais resistentes. Beta-lactamases de espectro estendido (ESBL) foram identificadas em 173 de 5.722 isolados Gram-negativos (3,0\%), 2010-2011. Conclusões: E. coli foi o isolado mais sensível a antibióticos. Houve uma evolução significativa da resistência antimicrobiana entre os dois períodos. Foi preocupante o aumento da resistência às fluoroquinolonas.

\section{ACKNOWLEDGEMENTS}

We would like to express gratitude to Valéria Cassettari, MD, MSc who is an infectious diseases physician and chief of the Infection Control Department at the University Hospital of the University of São Paulo (USP), whose work and dedication resulted in the database analyzed in this study under her authorization. Although she did not want to be part of writing this manuscript, her initiative was critical to this study and was only motivated by the spirit of the rational use of antibiotics and good clinical practice.

\section{CONFLICTS OF INTEREST (DISCLOSURES)}

No potential conflict of interest are declared for this paper.

\section{REFERENCES}

1. Andrade SS, Sader HS, Jones RN, Pereira AS, Pignatari AC, Gales AC. Increased resistance to first-line agents among bacterial pathogens isolated from urinary tract infections in Latin America: time for local guidelines? Mem Inst Oswaldo Cruz. 2006;101:741-8.

2. Bail L, Ito CAS, Esmerino LA. Infecção do trato urinário: comparação entre o perfil de susceptibilidade e a terapia empírica com antimicrobianos. Rev Bras Anal Clin. 2006;38:51-6

3. Bauer AW, Perry DM, Kirby WMM. Single disc antibiotic sensitivity testing of staphylococci; an analysis of technique and results. Arch Intern Med. 1959;104:20816.

4. Braoios A, Turatti TF, Meredija LCS, Campos TRS, Denadai FHM. Infecções do trato urinário em pacientes não hospitalizados: etiologia e padrão de resistência aos antimicrobianos. J Bras Patol Med Lab. 2009;45:449-56.

5. Clinical Laboratory Standards Institute. Normas de desempenho para teste de sensibilidade antimicrobiana: $15^{\circ}$ suplemento informativo. CLSI document M100-S15. Vol. 25 (1) Clinical Laboratory Standards Institute, Wayne, PA.

6. Clinical Laboratory Standards Institute. CLSI document M100-S16. Clinical Laboratory Standards Institute, Wayne, PA.

7. Clinical Laboratory Standards Institute. CLSI document M100-S20. Clinical Laboratory Standards Institute, Wayne, PA.

8. Clinical Laboratory Standards Institute. CLSI document M100-S21. Clinical Laboratory Standards Institute, Wayne, PA.

9. Costa LC, Belém LF, Freitas e Silva PM, Pereira HS, Silva Júnior ED, Leite TR, et al Infecções urinárias em pacientes ambulatoriais: prevalência e perfil de resistência aos antimicrobianos. Rev Bras Anal Clin. 2010;42:175-80 
PEIXOTO DE MIRANDA, E.J.; SALVADOR DE OLIVEIRA, G.S.; ROQUE, F.L.; DOS SANTOS, S.R.; OLMOS, R.D. \& LOTUFO, P.A. - Susceptibility to antibiotics in urinary tract infections in a secondary care setting from 2005-2006 and 2010-2011, in São Paulo, Brazil: data from 11,943 urine cultures. Rev. Inst. Med. Trop. Sao Paulo, 56(4): $313-24,2014$.

10. Dias RC, Marangoni DV, Smith SP, Alves EM, Pellegrino FL, Riley LW, et al. Clonal composition of Escherichia coli causing community-acquired urinary tract infections in the State of Rio de Janeiro, Brazil. Microb Drug Resist. 2009;15:303-8.

11. Dias VC, da Silva VL, Firmo Ede O, Bastos LQ, Bastos AN, Bastos RV, et al. Distribution of ESBL-producing enterobacteria associated to community-acquired monomicrobial urinary tract infections and antimicrobial susceptibility trends over a 9-year period. J Chemother. 2012;24:178-81.

12. Espinar MJ, Rocha R, Ribeiro M, Gonçalves Rodrigues A, Pina-Vaz C. Extended-spectrum $\beta$-lactamases of Escherichia coli and Klebsiella pneumoniae screened by the VITEK 2 system. J Med Microbiol. 2011;60:756-60.

13. Gales AC, Sader HS, Jones RN, SENTRY Participants Group (Latin America). Urinary tract infection trends in Latin American hospitals: report from the SENTRY antimicrobial surveillance program (1997-2000). Diagn Microbiol Infect Dis. 2002;44:289-99.

14. Gupta K, Hooton TM, Naber KG, Wullt B, Colgan R, Miller LG, et al. International clinical practice guidelines for the treatment of acute uncomplicated cystitis and pyelonephritis in women: a 2010 update by the Infectious Diseases Society of America and the European Society for Microbiology and Infectious Diseases. Clin Infect Dis. 2011;52:e103-20.

15. Hooton T. Clinical practice. Uncomplicated urinary tract infection. N Engl J Med. 2012;366:1028-37.

16. Hooton TM. The current management strategies for community-acquired urinary tract infection. Infect Dis Clin North Am. 2003;17:303-32.

17. Johnson L, Sabel A, Burman WJ, Everhart RM, Rome M, MacKenzie TD, et al. Emergence of fluoroquinolone resistance in outpatient urinary Escherichia coli isolates. Am J Med. 2008;121:876-84

18. Kiffer CR, Camargo EC, Shimakura SE, Ribeiro PJ Jr, Bailey TC, Pignatari AC, et al. A spatial approach for the epidemiology of antibiotic use and resistance in communitybased studies: the emergence of urban clusters of Escherichia coli quinolone resistance in São Paulo, Brazil. Int J Health Geogr. 2011;10:17
19. Kiffer CR, Mendes C, Oplustil CP, Sampaio JL. Antibiotic resistance and trend of urinary pathogens in general patients from a major urban city. Int Braz J Urol. 2007;33:42-9.

20. Koch CR, Ribeiro JC, Schnor OH, Zimmermann BS, Müller FM, D’ Agostin J, et al. Antimicrobial resistance of uropathogens among outpatients, 2000-2004. Rev Soc Bras Med Trop. 2008;41:277-81.

21. Lopes AA, Salgado K, Martinelli R, Rocha H. Aumento da freqüência de resistência à norfloxacina e ciprofloxacina em bactérias isoladas em uroculturas. Rev Assoc Med Bras. 1998;44:196-200

22. Mora JF, Menezes I, Requia MK, Sardiglia CU. Perfil dos pacientes com infecções do trato urinário diagnosticados no município de Flor do Sertão - SC. Rev Bras Anal Clin. 2008;40:321-3.

23. Nostrand JD, Junkins AD, Bartholdi RK. Poor predictive ability of urinalysis and microscopic examination to detect urinary tract infection. Am J Clin Pathol. 2000;113:709-13.

24. Poletto KQ, Reis C. Suscetibilidade antimicrobiana de uropatógenos em paciente ambulatoriais na Cidade de Goiânia, GO. Rev Soc Bras Med Trop. 2005;38:416-20.

25. Rocha JL, Tuon FF, Johnson JR. Sex, drugs, bugs, and age: rational selection of empirical therapy for outpatient urinary tract infection in an era of extensive antimicrobial resistance. Braz J Infect Dis. 2012;16:115-21.

26. Santo E, Salvador MM, Marin JM. Antimicrobial resistance among urinary tract Escherichia coli isolates from inpatients and outpatients in a tertiary care center in São Paulo, Brazil. Int J Infect Dis. 2007;11:558-9.

27. SIGTAP. Sistema de Gerenciamento da Tabela de Procedimentos. Available from: sigtap datasus.gov.br/

28. Stamm WE, Counts GW, Running KR, Fihn S, Turck M, Holmes KK. Diagnosis of coliform infection in acutely dysuric women. N Engl J Med. 1982;307:463-8.

Received: 15 August 2013

Accepted 13 November 2013 